Thrombosetherapie in der Onkologie

\section{Nierenfunktion beachten}

\author{
10-15\% aller Tumorpatienten \\ sind im Verlauf ihrer Erkrankung \\ von venösen Thromboembolien \\ (VTE) betroffen. Bei der Auswahl \\ einer Therapie der VTE muss \\ auch die Nierenfunktion berück- \\ sichtigt werden.
}

Nicht nur tumor-, sondern auch therapieassoziierte Faktoren führen zum erhöhten Thromboserisiko von Krebspatienten. So können die Art der Chemotherapie, die Behandlung mit antiangio- genen Substanzen oder supportive Maßnahmen wie ein zentraler Venenzugang das Risiko weiter steigern, erläuterte Florian Langer, Hamburg. In den Leitlinien [Farge D et al. Thromb Haemost. 2013;11(1):56-70] werden zur initialen Behandlung von Tumorpatienten mit einer VTE niedermolekulare Heparine $(\mathrm{NMH})$ empfohlen.

Auch bei der Erhaltungstherapie werden $\mathrm{NMH}$ einer Behandlung mit Vitamin-KAntagonisten vorgezogen. Bei der Auswahl eines bestimmten $\mathrm{NMH}$ ist die
Nierenfunktion zu berücksichtigen, was wegen des nephrotoxischen Potenzials vieler Chemotherapeutika und der häufig älteren Tumorpatienten in der Onkologie besonders relevant ist. NMH mit einem hohen Molekulargewicht sind weniger abhängig von der Nierenfunktion als kurzkettige. Das höchste Molekulargewicht der verfügbaren NMHs hat Tinzaparin (innohep ${ }^{\circledast}$ ) - es kann auch bei Patienten mit stark eingeschränkter Nierenfunktion eingesetzt werden [Siguret $\mathrm{V}$ et al. Thromb Haemost. 2000;84(5): 800-4].

Monika Walter

ONLEX ${ }^{\circledR}$ - 1. Onkologisches Leo ExpertenMeeting, Hamburg, 19.-20.04.2013; Veranstalter: LEO

Seit fünf Jahren steht Azacitidin (Vida$\mathrm{za}^{\bowtie}$ ) zur Therapie einer Hochrisiko-MDS zur Verfügung. Wie Lisa Pleyer, Salzburg, Österreich, berichtete, verzeichneten im österreichischen Register 66\% der Patienten nach dem ersten Einnahmezyklus das deutlichste Ansprechen, wobei sich das Ergebnis mit Fortsetzung der Therapie - auch bei allen anderen Patienten - vertiefte [Pleyer Letal. JHematolOncol.2013;6(1):32]. Die Therapie wurde insgesamt gut toleriert. Die häufigsten Grad-3/4 -Nebenwirkungen des betrafen hämatologische Toxizitäten (44,5\%), die aber das Gesamtüberleben nicht beeinflussten.

Friederike Klein les Giagounidis, Düsseldorf. Nach der MDS-004-Studie [Fennaux $\mathrm{P}$ et al. Blood. 2011;118(14):3765-76] habe die Therapie mit Lenalidomid (Revlimid ${ }^{\circledR}$ ) einen bedeutenden Effekt auf die Transfusionspflichtigkeit, erläuterte er. Unter der im Juni
2013 für diese Indikation zugelassenen Dosis von 10 mg erreichten $56 \%$ der Patienten die Transfusionsunabhängigkeit unter Placebo waren es nur $6 \%(\mathrm{p}<0,001)$. Dabei zeigte der Vergleich einer MDS-Kohorte, die mit Lenalidomid behandelt worden war, mit einer historischen Kohorte, dass unter der Therapie die Inzidenz einer AML nicht zunahm [Kuendgen A et al. Leukemia. 2013;27(5):1072-9].

\title{
Therapiechancen verlängern
}

\author{
Für Patienten mit intensiv vor- \\ behandeltem metastasierten \\ Kolorektalkarzinom (mCRC) wird \\ voraussichtlich noch 2013 der \\ Multikinase-Inhibitor Regorafenib \\ zugelassen.
}

Ergebnisse einer zulassungsrelevanten Phase-III-Studie belegen bei Patienten mit einem Rezidiv nach multipler Vorbehandlung eine längere Überlebenszeit unter Be- handlung mit dem Multikinase-Inhibitor Regorafenib (Stivarga ${ }^{\bowtie}$ ) im Vergleich zu Placebo [Grothey A et al. Lancet. 2013; 381(9863):303-12]. An der 2:1-randomisierten Doppelblindstudie nahmen 760 intensiv vorbehandelte mCRC-Patienten teil, die unter der Standardtherapie einen Tumorprogress erlitten hatten.

Im experimentellen Arm lag das Gesamtüberleben (OS) im Median mit 6,4 Monaten signifikant höher als im Place- boarm (5,0 Monate, Hazard Ratio [HR] 0,77; $\mathrm{p}=0,0052)$. Das Risiko einer Tumorprogression wurde um $51 \%$ gesenkt (medianes progressionsfreies Überleben [PFS] 1,9 vs. 1,7 Monate; HR 0,49; $\mathrm{p}=0,0001)$. Der OS- und PFS-Vorteil wurden sowohl für KRAS-Wildtyp als auch KRAS-mutierte Tumoren gezeigt. Die häufigsten Nebenwirkungen waren Hand-Fuß-Syndrom, Fatigue, Bluthochdruck, Diarrhö und Rash. IneSchmale

Media Akademie „Revitalizing Treatment Expectations in Colorectal Cancer", $15^{\text {th }}$ World Congress on Gastrointestinal Cancer (WCGC), Barcelona, Spanien, 03.07.2013; Veranstalter: Bayer Pharma 